\title{
Baseline Antibody Titre against Salmonella enterica in Healthy Population of Mumbai, Maharashtra, India
}

\author{
Rucha Patki, ${ }^{1}$ Sunil Lilani, ${ }^{2}$ and Dhaneshwar Lanjewar ${ }^{3}$ \\ ${ }^{1}$ Grant Government Medical College and Sir J. J. Group of Hospitals, Mumbai, Maharashtra, India \\ ${ }^{2}$ Shri Bhausaheb Hire Government Medical College and Hospital, Dhule, India \\ ${ }^{3}$ Department of Pathology, Grant Government Medical College and Sir J. J. Group of Hospitals, Mumbai, Maharashtra, India
}

Correspondence should be addressed to Rucha Patki; ruchaapatki@gmail.com

Received 10 May 2017; Accepted 3 July 2017; Published 31 August 2017

Academic Editor: Barbara H. Iglewski

Copyright ( 2017 Rucha Patki et al. This is an open access article distributed under the Creative Commons Attribution License, which permits unrestricted use, distribution, and reproduction in any medium, provided the original work is properly cited.

\begin{abstract}
Objective. The aim of this study was to establish a baseline titre for the population of Mumbai, Maharashtra, India. Method. Four hundred healthy blood donors, attending blood donation camps, were screened using a survey questionnaire. Widal tube agglutination test was performed on the diluted sera (with $0.9 \%$ normal saline) of blood donors, with final dilution ranging from $1: 40$ to $1: 320$. Results. Out of 400 individuals providing samples, 78 (19.5\%) individuals showed antibody titres $\geq 1: 40$ for at least one antigen and $322(80.5 \%)$ showed no agglutination. The baseline antibody titres against $\mathrm{O}$ antigen and $\mathrm{H}$ antigen of Salmonella enterica serotype Typhi were found to be 1:40 and 1:80, respectively. Similarly, the baseline antibody titres for the $\mathrm{H}$ antigen of Salmonella enterica serotypes Paratyphi A and Paratyphi B were found to be $1: 40$ and 1:80, respectively. Conclusion. Thus, it was noted that the diagnostically significant cutoff of antibody titre from acute phase sample was $\geq 1: 80$ for $S$. Typhi $O$ antigen and titre of $\geq 1: 160$ for both $S$. Typhi H antigen and $S$. Paratyphi BH antigen. Antibody titre of $\geq 1: 80$ can be considered significant for $S$. Paratyphi AH antigen.
\end{abstract}

\section{Introduction}

The term enteric fever includes typhoid fever, caused by Salmonella enterica subspecies enterica serotype Typhi and paratyphoid fever caused by Salmonella enterica subspecies enterica serotypes Paratyphi A, Paratyphi B, and Paratyphi C [1]. Endemic in India, there are an estimated 6,345,776 cases per year of enteric fever with case fatality rate $<1 \%$ [2]. The criterion standard for diagnosis of enteric fever has long been culture isolation of the organism [3]. Among these, the isolation of bacteria from blood or bone marrow is considered the gold standard. However, in developing countries, culture facilities are not easily available or accessible and serological tests such as Widal test remain the mainstay of diagnosis. The Widal test detects the presence of antibodies against antigens of Salmonella enterica in the blood. However, in healthy populations of the endemic areas, the antibody titre may be found to be high in the presence of cross-reacting antigens owing to other infections, a healthy carrier state, long-standing liver disease, and previous typhoid vaccination [4]. Therefore, it becomes necessary to collect sera from an acute phase and a convalescent phase, at least 10-14 days apart to demonstrate a fourfold rise in the antibody titres [5]. However, a rise in the titre of antibodies cannot always be substantiated on account of early rise in antibodies due to high endemicity, late collection of samples in the natural history of disease, or prior intake of antibiotics [6-8]. Furthermore, an anamnestic response may develop during an acute febrile illness, such as malaria, in people with past Salmonella infection or history of vaccination against Salmonella [1]. In order to use the Widal test effectively, it is required that an accurate baseline titre is obtained for the diagnosis of enteric fever, so as to interpret the antibody titre results from a single acute phase sample against the baseline Widal titre present in healthy individuals in that particular geographical area $[8,9]$. Substantial differences in baseline Widal titres are present in healthy individuals of different geographical areas, depending on the endemicity of enteric fever in those areas, as determined by studies done in other states of India such as Karnataka, Uttarakhand, Gujarat, and Kerala [10-13]. 
The baseline antibody titre has not been established for the Mumbai region. In view of the above, this study was carried out to ascertain the baseline antibody titre, in the geographical area of Mumbai city of Maharashtra state, India, taking into consideration the fact that enteric fever is a major public health problem in Mumbai, India.

\section{Materials and Methods}

This study was a cross-sectional type of study carried out at the Department of Microbiology, Grant Government Medical College and Sir J. J. Group of Hospitals, Mumbai, Maharashtra, India, after obtaining an approval from the Institutional Ethics Committee. Blood samples were collected after obtaining written informed consent from healthy blood donors $(N=400)$, of both genders and those aged 18 to 55 years, who attended the various blood donation camps organized by the institution during the period extending from May 2014 to September 2014.

The blood donors were screened using a survey questionnaire. Blood samples were collected from apparently healthy donors, who had neither been vaccinated with TAB (Typhoid and Paratyphoid A and B) vaccine or oral typhoid vaccine/Vi vaccine nor had suffered from enteric fever in the past. We selected only those blood donors who were permanent residents of Mumbai, India.

Individuals with any active or recent infections including hepatitis B, hepatitis C, malaria, Dengue fever, brucellosis, and HIV/AIDS and any type of fever in the last 6 months were excluded from this study.

A venous blood sample of $5 \mathrm{ml}$ was collected in a plain tube and was allowed to clot at room temperature for about 30 minutes. The samples were then centrifuged at $3000 \mathrm{rpm}$ (revolutions per minute) for 10 minutes in order to separate the serum from the blood. Sera were transferred to clean and dry sterile storage vials. The serum samples were refrigerated at $-20^{\circ} \mathrm{C}$. Stained Salmonella antigen test kit for tube test was provided by Star Diagnostics Pvt. Ltd., India. This test kit contained smooth suspensions of antigens "O" and " $\mathrm{H}$ " of Salmonella serotype Typhi, " $\mathrm{H}$ " antigen of Salmonella serotype Paratyphi A, and " $\mathrm{H}$ " antigen of Salmonella serotype Paratyphi B.

The test was performed after bringing sera and reagents to room temperature. Widal titre was estimated by confirmatory quantitative tube agglutination test using standard agglutination test procedure [4]. As per the manufacturer's instructions, $0.5 \mathrm{ml}$ of the increasingly diluted sera, dilutions ranging from $1: 20$ to $1: 320$ in $0.9 \%$ normal saline, was tested by adding an equal amount $(0.5 \mathrm{ml})$ of antigen, thereby giving final dilution ranging from $1: 40$ to $1: 640$, and the tubes were then incubated overnight at $37^{\circ} \mathrm{C}$ in a water bath. Saline and positive controls were added in every set of the test. The tubes were mixed well and incubated in a serological water bath maintained at $37^{\circ} \mathrm{C}$ for $16-20$ hours and were observed for agglutination. In a positive test, $\mathrm{O}$ antigen showed granular agglutination and $\mathrm{H}$ antigen showed fluffy agglutination. The last tube showing visible agglutination with the naked eye was taken as an endpoint of the test. The titre was reported out
TABLE 1: Widal test results.

\begin{tabular}{lc}
\hline Widal status & Number of individuals $(N=400)$ \\
\hline AB titre $\geq 1: 40$ & $78(19.50 \%)$ \\
AB titre $<1: 40$ & $322(80.50 \%)$
\end{tabular}

$N$ : total number of participant blood donors; $\mathrm{AB}$ : antibody.

TABLE 2: Distribution of positive samples (antibody titre $\geq 1: 40$ ) against different Salmonella serotypes.

\begin{tabular}{lcc}
\hline Serotype & Antibody type & $\begin{array}{c}\text { Positive results }(N=400) \\
n(\%)\end{array}$ \\
\hline S. Typhi & Anti-TO & $37(9.25 \%)$ \\
S. Typhi & Anti-TH & $41(10.25 \%)$ \\
S. Paratyphi A & Anti-TH & $2(0.50 \%)$ \\
S. Paratyphi B & Anti-BH & $15(3.75 \%)$ \\
\hline
\end{tabular}

$N$ : total number of participant blood donors; $n$ : number of individuals with antibody titre $\geq 1: 40$ for respective serotype.

as the reciprocal of the endpoint. Absence of agglutination suggested a negative test.

The method used for finding the baseline titre was Widal tube agglutination test. The amount of antigen suspension used was the same as the amount of diluted sera making the dilution 2-fold, and hence the readings in our study begin with titre $1: 40$, which is in accordance with the manufacturer's instructions and also the standard lab protocol followed by the Department of Microbiology of the institution where our study was conducted.

2.1. Statistical Analysis. Data was analyzed in an Excel sheet and the variables were summarized using frequency count and percentage.

\section{Results}

A total of 400 apparently healthy blood donors provided serum samples for testing the baseline antibody titre against Salmonella enterica, using Widal tube agglutination test. Blood donors were in the age group of 18 to 55 years. Out of all participant blood donors, $352(88 \%)$ were males. Out of the 400 individuals, 78 individuals $(19.5 \%)$ were found to be positive $(\geq 1: 40)$ for at least one of the antigens. 322 individuals $(80.5 \%)$ were found to be negative $(<1: 40)$ for all antigens (Table 1).

As seen in Table 2, among 400 individuals, a total of 37 $(9.25 \%)$ individuals were found positive $(\geq 1: 40)$ for $S$. Typhi "O" antigen and $41(10.25 \%)$ were found positive $(\geq 1: 40)$ for $S$. Typhi " $H$ " antigen. Only 2 (0.5\%) individuals were positive $(\geq 1: 40)$ for $S$. Paratyphi AH antigen. The total number of individuals positive $(\geq 1: 40)$ for $S$. Paratyphi $\mathrm{BH}$ antigen was $15(3.75 \%)$.

As seen in Table 3, among the 37 (9.25\%) individuals who were found to be positive against $\mathrm{O}$ antigen of $S$. Typhi, 20 (5\%) individuals had a titre of $1: 40,14$ (3.5\%) individuals had a titre of $1: 80,1(0.25 \%)$ individual had a titre of $1: 160$, and 2 $(0.5 \%)$ individuals had a titre of $1: 320$. Similarly, among the total of $41(10.25 \%)$ individuals positive against $\mathrm{H}$ antigen of 
TABLE 3: Comparison of frequency and percentage of baseline antibody titres against different serotypes of Salmonella enterica.

\begin{tabular}{|c|c|c|c|c|c|}
\hline \multirow{2}{*}{ Antigen } & \multirow{2}{*}{ Total } & \multicolumn{4}{|c|}{ Dilution end titres $(N=400)$} \\
\hline & & $1: 40$ & $1: 80$ & $1: 160$ & $1: 320$ \\
\hline S. Typhi "O” & $37(9.25 \%)$ & $20(5 \%)$ & $14(3.5 \%)$ & $1(0.25 \%)$ & $2(0.5 \%)$ \\
\hline S. Typhi "H” & $41(10.25 \%)$ & $11(2.75 \%)$ & $25(6.25 \%)$ & $5(1.25 \%)$ & - \\
\hline S. Paratyphi "AH" & $2(0.5 \%)$ & $1(0.25 \%)$ & $1(0.25 \%)$ & - & - \\
\hline S. Paratyphi "BH" & $15(3.75 \%)$ & $4(1 \%)$ & $7(1.75 \%)$ & $4(1 \%)$ & - \\
\hline
\end{tabular}

$N$ : total number of participant blood donors.

S. Typhi, 11 (2.75\%) individuals had a titre of 1: 40, $25(6.25 \%)$ individuals had a titre of $1: 80$, and 5 (1.25\%) individuals had a higher titre of $1: 160$.

As seen in Table 3, out of the $2(0.5 \%)$ individuals who tested positive against $\mathrm{H}$ antigen of S. Paratyphi A, $1(0.25 \%)$ individual had a titre of $1: 40$ and the other individual had a titre of $1: 80$. It can be seen that very few individuals $(0.5 \%)$ were positive for $\mathrm{H}$ antigen of $S$. Paratyphi A.

Among 15 (3.75\%) individuals positive against $\mathrm{H}$ antigen of S. Paratyphi B, $4(1 \%)$ individuals had a titre of $1: 40,7$ $(1.75 \%)$ had a titre of $1: 80$, and only $4(1 \%)$ samples reported a higher titre of $1: 160$ (Table 3 ).

\section{Discussion}

Isolation of Salmonella from blood or bone marrow has always been the gold standard for diagnosis of enteric fever. However, most healthcare centers in India do not have easy access to this method and also culture methods are seen as time-consuming. The Widal agglutination test has always been the preferred test for diagnosis of enteric fever in many developing countries, including India, where it is extensively practiced. Especially in endemic areas, the Widal test is still of significant diagnostic value, provided judicious interpretation of the test is made against a background of pertinent information, especially data which relate to agglutinin levels in normal individuals and in nontyphoidal fevers common in the region [8].

This study was the first ever study done in Mumbai, to the best of our knowledge, which was conducted for the estimation of baseline antibody titre in healthy individuals against serotypes Typhi and Paratyphi A and Paratyphi B of Salmonella enterica. Our study has shown that disparate titres of antibody, against the different serotypes of Salmonella enterica, exist in healthy individuals.

The results of our study established the presence of high antibody titres in healthy individuals. Out of 78 (19.5\%) healthy individuals with positive titres for at least one antigen, we found that $37(9.25 \%)$ had anti-O antibody titres of $\geq$ $1: 40$ and $41(10.25 \%)$ had antibody titres of $\geq 1: 80$ against $S$. Typhi $\mathrm{H}$ antigen. Taking these results into consideration, the baseline antibody titres against $\mathrm{O}$ antigen and $\mathrm{H}$ antigen of Salmonella enterica serotype Typhi were found to be $1: 40$ and $1: 80$, respectively. Our results are in concordance with those reported in studies conducted by researchers in other states of India that the disease is endemic to, such as Karnataka and Uttarakhand, where the recorded baseline titres against $\mathrm{O}$ antigen and $\mathrm{H}$ antigen of $S$. Typhi were found to be $1: 40$ and
$1: 80$, respectively, in both states $[10,11]$. A similar study was done in Nepal by Pokhrel et al., which was the first study done in Nepal to determine the baseline titre and cutoff values for the diagnosis of enteric fever. It was concluded that significant titres are those higher than 1:80 for anti-O and higher than 1:160 for anti-H antigen of Salmonella enterica serotype Typhi, for a possible diagnosis of typhoid fever for Kathmandu Valley, Nepal [14].

In this study, the greatest anti- $\mathrm{O}$ and anti- $\mathrm{H}$ titres against $S$. Typhi were $1: 320$ and $1: 160$ in $0.5 \%$ and $1.25 \%$, respectively. Such higher titres of $1: 160$ against $O$ and $H$ antigens of $S$. Typhi were seen in a study carried out in Kerala, India, by Sreenath [13]. We obtained a very low number of individuals showing antibody titres against $\mathrm{H}$ antigen of $S$. Paratyphi A. This study demonstrated that anti-H agglutinin titre to Salmonella enterica serotypes Paratyphi A and Paratyphi B in Mumbai was lower than that against Salmonella enterica serotype Typhi. A total of 15 individuals had anti-H titre $\geq$ 1: 40 to Salmonella enterica serotype Paratyphi B. A very little proportion of individuals showed significant levels of anti-H titre against Salmonella enterica serotype Paratyphi A, indicating that it is not a usual serotype in Mumbai.

Widal agglutination test can be positive in individuals with previous immunization against Salmonella antigen, infection with other Enterobacteriaceae, and other diseases such as malaria and Dengue fever and also due to variability and poor standardization of commercial antigen preparation. False positive agglutination can also be seen as a cross reaction with nontyphoidal Salmonella [15]. A study conducted in Papua New Guinea by Clegg et al. was able to clearly demonstrate a remarkable change in the immune status of the community, in which those healthy individuals with a Widal tube $\mathrm{O}$ agglutination titre of 40 or more rose from 0 to $56 \%$ in the short span of five years. It was found that the factors influencing the usefulness of the Widal agglutination test in a population include the endemicity of typhoid fever, the immune response to typhoid, baseline antibody levels of the general population to Salmonella Typhi, and the ability to select an appropriate diagnostic cutoff titre [16]. Though its usefulness will vary with time and place, the Widal agglutination test remains a useful diagnostic test under appropriate circumstances. Hence, establishing and updating the baseline Widal titre for a particular area are an important factor in the proper interpretation of the Widal test. Thus, assessment of the Widal baseline titre for healthy individuals in a specific geographical area should be performed from time to time.

Various studies which have been carried out in different states of India have shown that the baseline titre is different 
in different geographical locations. Hence, the baseline titre of a specified geographical area should first be established [1012]. Typhoid fever remains a major public health problem in developing countries like India [17]. Being inexpensive and readily available, Widal tube agglutination test will probably remain the preferred diagnostic test in many developing countries, such as ours, understanding that the baseline antibody titre is established.

\section{Conclusions}

Widal test results from an acute phase serum sample should be interpreted based on the baseline titre prevalent in healthy individuals in that particular geographic area. In this study, the baseline antibody titres against $\mathrm{O}$ antigen and $\mathrm{H}$ antigen of Salmonella enterica serotype Typhi were found to be 1:40 and $1: 80$, respectively. Similarly, the baseline antibody titres for the $\mathrm{H}$ antigen of Salmonella enterica serotype Paratyphi A and Salmonella enterica serotype Paratyphi B were found to be $1: 40$ and $1: 80$, respectively. Thus, an antibody titre, from acute phase sample, of $\geq 1: 80$ for $S$. Typhi $O$ antigen and titre of $\geq 1: 160$ for both $S$. Typhi $\mathrm{H}$ and $S$. Paratyphi B H antigens and antibody titre of $\geq 1: 80$ for $\mathrm{H}$ antigen of $S$. Paratyphi A can be considered as diagnostically significant.

\section{Disclosure}

This study was presented at the Leiden International Medical Students Conference, held in Leiden, Netherlands (LIMSC2015).

\section{Conflicts of Interest}

The authors declare that they have no conflicts of interest.

\section{Acknowledgments}

The authors are grateful to the Indian Council of Medical research (ICMR) for funding this study with the grant awarded for the STS project. They would also like to thank Dr. Abhay Chowdhary, HOD, Department of Microbiology, Grant Govt. Medical College, Mumbai; Dr. Shilpa Patil, Professor, Department of Microbiology, Grant Govt. Medical College, Mumbai; and Ms. Sonal More for providing constant support and guidance throughout the study.

\section{References}

[1] R. Ananthanarayan and C. K. J. Paniker, Textbook of Microbiology, Universities Press, Hyderabad, India, 9th edition, 2013.

[2] J. Dave, M. Millar, H. Maxeiner et al., "East London experience with enteric fever 2007-2012," PLoS ONE, vol. 10, no. 3, Article ID e0120926, 2015.

[3] 1. Dan, S. Lango Anthony, L. Dennis Kasper, L. Stephen, and J. Larry Jameson, Harrison's Principle of Internal Medicine, vol. 1276, 18th edition, 2012.

[4] J. G. Colle, A. G. Frason, B. P. Marmion, and A. Simmons, Mackie and Mc Cartney Practical Medical Microbiology, vol. 38, Churchill Livingstone, New York, NY, USA, 1996.
[5] A. Ismail, "New advances in the diagnosis of typhoid and detection of typhoid carriers," The Malaysian Journal of Medical Sciences: MJMS, vol. 7, no. 2, pp. 3-8, 2000.

[6] M. Chessbrough, Medical Laboratory Manual for Tropical Countries, ELBS, Cambridge, 2nd edition, 1987.

[7] C. M. Parry, L. Wijedoru, A. Arjyal, and S. Baker, "The utility of diagnostic tests for enteric fever in endemic locations," Expert Review of Anti-Infective Therapy, vol. 9, no. 6, pp. 711-725, 2011.

[8] T. Pang and S. D. Puthucheary, "Significance and value of the Widal test in the diagnosis of typhoid fever in an endemic area," Journal of Clinical Pathology, vol. 36, no. 4, pp. 471-475, 1983.

[9] A. Willke, O. Ergonul, and B. Bayar, "Widal test in diagnosis of typhoid fever in Turkey," Clinical and Diagnostic Laboratory Immunology, vol. 9, no. 4, pp. 938-941, 2002.

[10] N. S. Madhusudhan and A. H. Manjunath, "Determination of baseline widal titre among healthy population," International Journal of Biomedical Research, vol. 3, no. 12, pp. 437-438, 2012.

[11] S. Pal, R. Prakash, D. Juyal, N. Sharma, A. Rana, and S. Negi, "The baseline Widal titre among the healthy individuals of the hilly areas in the Garhwal region of Uttarakhand, India," Journal of Clinical and Diagnostic Research, vol. 7, no. 3, pp. 437-440, 2013.

[12] J. M. Payal and J. Binita, "Aring baseline antibody titre against salmonella enterica in healthy population of gujarat, India," International Journal of Scientific Research, vol. II, no. V, pp. 493494, May 2013.

[13] K. Sreenath, "Detection of baseline Widal titres among the blood donors: A population," in Detection of baseline Widal titres among the blood donors: A population basedstudy.Int. J.Curr.Microbiol.App.Sci.2014.3:428-431, pp. 428-431.

[14] B. M. Pokhrel, R. Karmacharya, S. K. Mishra, and J. Koirala, "Distribution of antibody titer against Salmonella enterica among healthy individuals in nepal," Annals of Clinical Microbiology and Antimicrobials, vol. 8, article no. 1, 2009.

[15] L. A. Olopoenia and A. L. King, "Widal agglutination test - 100 years later: Still plagued by controversy," Postgraduate Medical Journal, vol. 76, no. 892, pp. 80-84, 2000.

[16] A. Clegg, M. Passey, M. Omena, K. Karigifa, and N. Suve, "Reevaluation of the widal agglutination test in response to the changing pattern of typhoid fever in the highlands of Papua New Guinea," Acta Tropica, vol. 57, no. 4, pp. 255-263, 1994.

[17] R. L. Ochiai, C. J. Acosta, M. C. Danovaro-Holliday et al., "A study of typhoid fever in five Asian countries: disease burden and implications for controls," Bulletin of the World Health Organization, vol. 86, no. 4, pp. 260-268, 2008. 

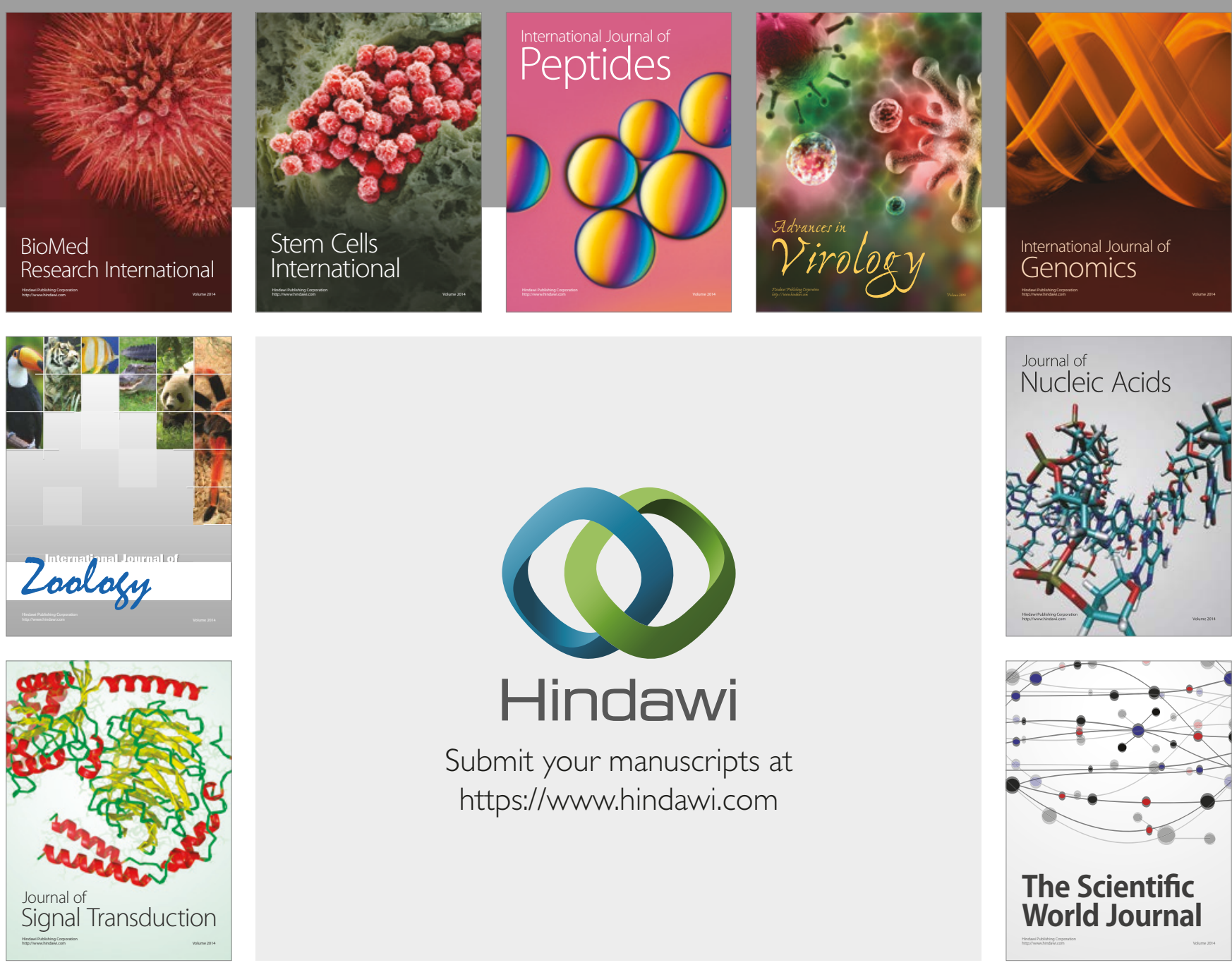

Submit your manuscripts at

https://www.hindawi.com
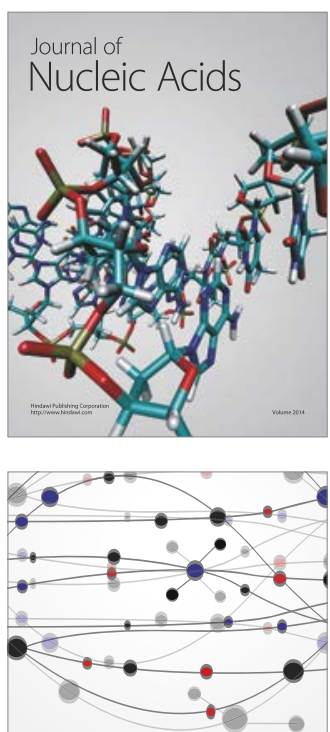

The Scientific World Journal

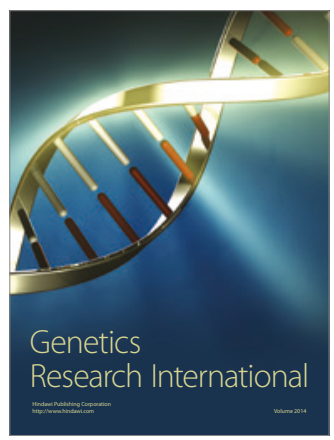

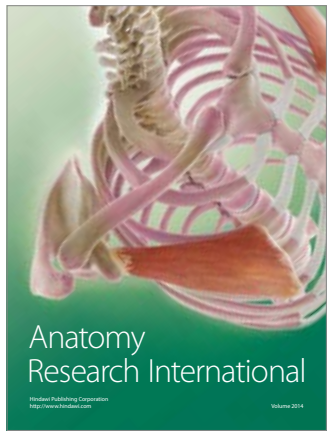

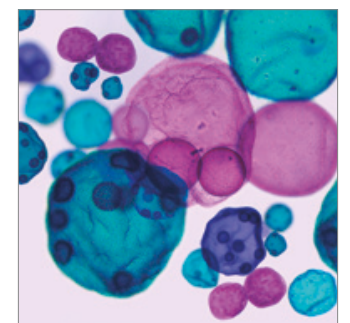

International Journal of Microbiology
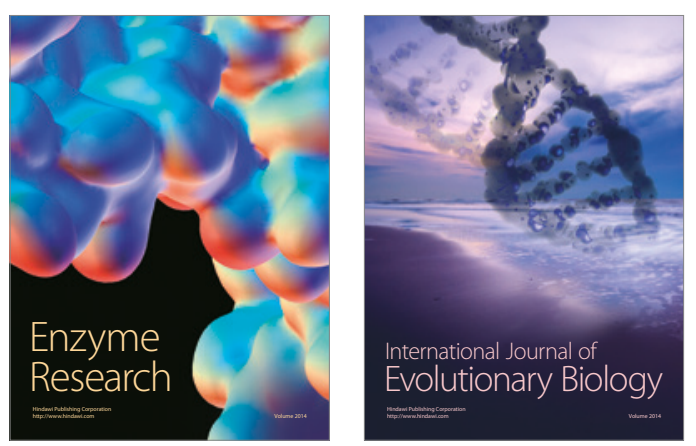
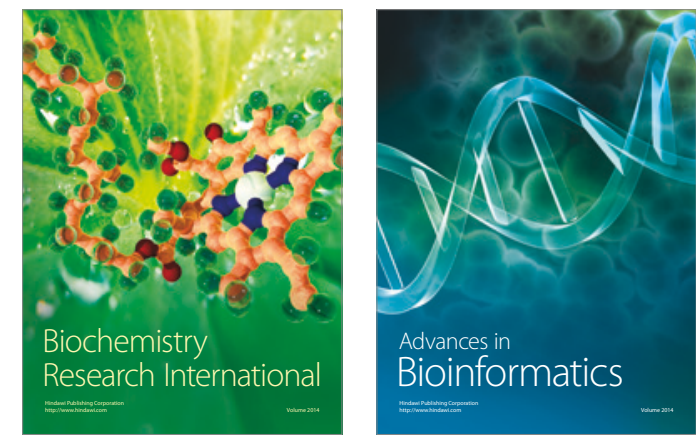

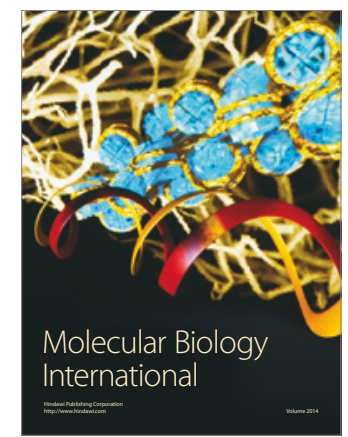

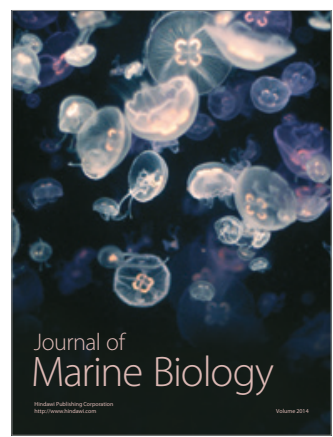

